\title{
Is ambulatory management of primary spontaneous pneumothorax safe and effective?
}

\author{
Audrey Marcotte $^{1}$ (D) . François de Champlain ${ }^{2}$
}

Received: 14 May 2021 / Accepted: 19 July 2021 / Published online: 6 August 2021

(c) The Author(s), under exclusive licence to Canadian Association of Emergency Physicians (CAEP)/ Association Canadienne de Médecine d'Urgence (ACMU) 2021

Keywords Pneumothorax $\cdot$ Chest tube

Full Citation: Hallifax RJ, McKeown E, Sivakumar P, Fairbairn I, Peter C, Leitch A, et al. Ambulatory management of primary spontaneous pneumothorax: an openlabel, randomised controlled trial. The Lancet. 2020 Jul $4 ; 396(10243): 39-49$

Abstract Link: https://www.sciencedirect.com/science/ article/pii/S0140673620310436

Article Type: Therapy, Randomized Controlled Trial

Ratings: Methods—4/5, Usefulness-3/5

\section{Introduction}

\section{Background}

The optimal management for primary spontaneous pneumothorax is not well defined and may lead to unnecessary hospitalizations.

\section{Objectives}

To describe the duration of hospitalization and safety of ambulatory management of primary spontaneous pneumothorax compared with standard care.

Audrey Marcotte

Audrey.Marcotte@mail.mcgill.ca

1 Emergency Medicine Residency Program, McGill University, McGill University Health Center, Montreal, QC, Canada

2 Department of Emergency Medicine, McGill University, McGill University Health Center, Montreal, QC, Canada

\section{Methods}

\section{Design}

Open-label randomized controlled trial.

\section{Eligibility criteria}

Adults (aged 16-55) with symptomatic, clinically stable primary spontaneous pneumothorax.

\section{Intervention}

Insertion of an ambulatory device-Rocket pleural vent, an $8 \mathrm{~F}$ gauge catheter attached to a self-contained, one way Heimlich valve and fluid collection chamber.

\section{Comparison}

Standard care—aspiration, chest tube insertion, or both.

\section{Outcomes}

The primary outcome was total length of hospital stay including re-admission up to 30 days after randomization. Secondary outcomes were need for a further pleural procedure, adverse events, pain and breathless scores, recurrence rates, and time off work because of pneumothorax treatments.

\section{Main results}

236 patients were enrolled; 117 were assigned to ambulatory care and 119 to standard care. The mean age was 30 years (SD 8), and the majority were male ( $82 \%)$. The 
median hospital stay at 30 days was significantly lower for the ambulatory care group (mean difference, 2 days, 95\% CI 1-3). Time until successful completion of treatment (defined as adequate lung expansion following needle aspiration or removal of device or chest tube) was significantly longer in the ambulatory care group (mean difference -1 days, $95 \% \mathrm{CI}$ -2 to $0, p=0.0040$ ). Fewer additional pleural procedures were required in those who received ambulatory care $(21 \%$ vs. $35 \% p=0.0075$ ). However, more adverse events occurred in the ambulatory group including enlarging pneumothorax, blocked, dislodged or leaking device, and asymptomatic re-expansion pulmonary edema (55\% vs. $39 \%, p=0.0135$ ). Re-admission rates, time off work, and mean pain, breathlessness scores, and analgesia use were similar.

\section{Appraisal}

\section{Strengths}

- Clear question that challenges current practice for primary spontaneous pneumothorax.

- Adherence to CONSORT guidelines.

- Evaluation of patient-centered outcomes.

- Pragmatic multicenter RCT design with sensitivity analysis and excellent follow-up.

\section{Limitations}

- Mandatory open label.

- Recruitment bias-patients only recruited during daytime hours when research assistant was present.

- Needle aspiration in the standard care arm was left up to the local physician and not performed in $26 \%$ of the time which may have introduced bias. However, a post hoc analysis of the primary outcome excluding patients in whom no aspiration was done still favored the intervention arm.

- Use of single commercial ambulatory device may limit generalizability to other devices or chest tube with Heimlich valve.

- Follow-up requirements used for the ambulatory care group to detect adverse events may be difficult to implement in a real-life setting.

\section{Context}

The American College of Chest Physicians considers ambulatory treatment with a small-bore catheter and Heimlich valve a reasonable option for clinically stable, reliable patients with large pneumothorax and evidence of re-expansion [1]. Canadian recommendations do not exist and British guidelines do not suggest an ambulatory care option [2]. Canadian practice is highly variable with some thoracic specialists already managing this population as outpatients based on low quality evidence [3]. Our thoracic surgeons suggest that this study reflects their experience of successful outpatient management and few adverse events; however, warn that the specific ambulatory device type and insertion location may impact success of this treatment option.

\section{Bottom line}

This study demonstrates that outpatient treatment with an ambulatory device of clinically stable patients with primary spontaneous pneumothorax is safe and effective at reducing the hospital length of stay and the required number of pleural procedures.

However, ambulatory treatment options necessitate outpatient care pathways in collaboration with surgical consultants to ensure appropriate detection of adverse events and timely removal of the device. Guidelines should be updated to reflect this option and emphasize a shared decision-making process to highlight the importance of adequate followup and the risk of adverse events.

\section{Declarations}

Conflict of interest No conflicts of interest to declare from either author.

\section{References}

1. Baumann MH, Strange C, Heffner JE, Light R, Kirby TJ, Klein J, et al. Management of spontaneous pneumothorax: an American college of chest physicians Delphi consensus statement. Chest. 2001;119(2):590-602.

2. MacDuff A, Arnold A, Harvey J. Management of spontaneous pneumothorax: British Thoracic Society pleural disease guideline 2010. Thorax. 2010;65(Suppl 2):18-31.

3. Brims FJH, Maskell NA. Ambulatory treatment in the management of pneumothorax: a systematic review of the literature. Thorax. 2013;68(7):664-9. 